\title{
ABSTRACTS \\ 10th Annual Evidence-Based Practice/ Research Conference \\ Evidence for Practice: Origins and New Directions October 8, 2018
}

General Session Abstracts

1.1-3.2

Poster Abstracts

P1-P34 


\section{1 "Susie" Pet Robotic Therapy in Acute Care Hospital Settings}

Penny Huddleston, PhD, RN, NEA-BC, CCRN; Dorothy Dawes, MSN/MHA, RN, NE-BC; Johna Gilbert, ADN, RN; Beverly Colbert, BSN, RN, CCRN; Brenda Fine, BSN, RN, CCRN; Linda Tjiong, MSN, DBA, RN, NE-BC; Melissa Winter, DNP, RN, NEA-BC; Linda Denke, PhD, RN; Dawn Graham, BS, RN, CVN

Baylor Scott \& White Medical Center, Irving, TX

Baylor Scott \& White Medical Center, McKinney, TX

Background: Evidence indicated that use of the PARO robotic pet reduced difficult behavioral and psychological symptoms with patients diagnosed with dementia; however, there was no evidence found in using the PARO robotic pet in adult patients with behavioral disturbances in the acute care setting.

Methods: The Pittsburgh Agitation Scale (PAS) and Zung Self-Rating Anxiety Scale (Zung) along with physiologic measures of heart rate (HR), respiratory rate (RR), blood pressure (BP), and pulse oximeter (SPO2) readings before and after the treatment of the PARO in the experimental group vs listening to the television or music in the control group were used to determine if agitation and/or anxiety could be decreased in 40 subjects at two facilities in the Baylor Scott \& White Health System.

Results: Comparison of all measures, whether physiological (HR, RR, BP, SPO2), agitation (PAS), or anxiety (Zung), showed no statistically significant contrasts at the $95 \%$ confidence level with $P$ value set at 0.05 between the experimental and control groups for the PAS test between before and after relaxation, or between exposure episodes before or after relaxation method; however, a clinically significant lowering of the distributional location of the Zung anxiety score was noted in the experimental group after exposure to the relaxation method.

Conclusion: Based on the results, further research needs to be conducted with the use of the PARO to determine if the robotic seal will reduce agitation and anxiety in patients in the acute care hospital setting. Further research with a larger sample size and in more than one healthcare system is necessary to demonstrate the statistical significance that the use of the PARO reduces anxiety and agitation in this patient population.

\subsection{A. Smoking in Pregnancy in Indigenous Women: Not Simply Just a Matter of Choice}

Sandra Small, PhD, RN; Cindy Murray, PhD, RN; Caroline Porr, PhD, RN

Memorial University of Newfoundland, St. John's, Newfoundland

Background: Although the overall prevalence of smoking among pregnant women has declined in recent years, rates of smoking during pregnancy among indigenous women remain alarmingly high. We conducted a systematic review of qualitative evidence to understand the experiences and cessation needs of indigenous women internationally who smoked during pregnancy. We found a small body of research evidence with confidence in the synthesized findings determined to be low to very low due largely to methodological limitations among the exclusively qualitative descriptive studies. Hence, we are conducting a study about indigenous women's experiences of smoking during pregnancy to contribute further to the evidence.

Methods: We are using phenomenology based on a recommendation in our systematic review that future studies about smoking in pregnant indigenous women employ rigorous methodologies to strengthen understanding. We have completed 26 in-depth interviews with 15 indigenous women who smoked during pregnancy and have analyzed the interviews for themes. We plan to interview up to 5 more indigenous women to finalize the themes. The study, which received research ethics approval, is taking place in Newfoundland and Labrador, Canada in collaboration with an indigenous organization.

Results: The preliminary findings reveal that the women had different experiences: quitting smoking during pregnancy for baby's health; quitting smoking during pregnancy because of adverse physiological effects; continuing to smoke during pregnancy but remorseful and wanting to quit; continuing to smoke during pregnancy and resigned to the addiction; and continuing to smoke during pregnancy as it is their personal choice. The women were on their own in their experience of smoking and quitting during pregnancy as they had inadequate support from their social network and healthcare providers.

Conclusion: The findings of this study are consistent with the findings of the systematic review. It is recommended that healthcare providers take every opportunity to offer evidence-based smoking cessation interventions and ongoing support to pregnant indigenous women. 


\title{
2.1.B Improving Blood Glucose Control in Non-Critically III Hospitalized Adults by Reducing Repeat Hyperglycemic Episodes
}

\author{
Stacey Seggelke, DNP, RN \\ University of Colorado, Denver, CO
}

Background: Hyperglycemia occurs in a significant proportion of hospitalized patients and contributes to increased morbidity, mortality, and length of stay (LOS). Despite knowledge of the importance of glycemic control, this target is not being achieved, with hyperglycemia occurring in approximately $40 \%$ of all hospitalizations on two inpatient units.

Methods: A quality improvement protocol was developed to reduce repeat hyperglycemia, defined as blood glucose level $>180 \mathrm{mg} / \mathrm{dL}$ on two or more consecutive days, as the primary outcome and reduced time with hyperglycemia during LOS as the secondary outcome. The process measures included were education, referrals to an inpatient glucose management team, and the use of an in-room blood glucose log. The balance measure was the rate of hypoglycemia on two medical units.

Results: Preintervention and postintervention measures of repeat hyperglycemia improved from $>40$ to $<25 \%$ on one medical unit. No improvement was seen in time spent with hyperglycemia during LOS. Referrals to the inpatient glucose management increased from $46 \%$ to $58 \%$ on one unit, and education increased nursing knowledge for inpatient glycemic targets on both units.

Conclusion: Quality improvement interventions led to an improvement in repeat hyperglycemia in hospitalized adults.

\subsection{A. Families Without Mothers: Exploring Life After Maternal Death}

Donald Johnston, PhD, RN-MHS, RRT; Patty Atkins, MSN, RN

Northwestern State University, Shreveport, LA

Background: This study sought to uncover a previously unexplored aspect of maternal death: the stories of children and families left behind. By replacing numbers with stories, we sought to personalize the problem, raise awareness, and identify needs. The study sought to answer the following questions: What happens to the children left behind after maternal death? What is the impact on the surviving family and on the community as a whole? What kinds of support are most meaningful to survivors?

Methods: Using interpretive phenomenological analysis, we explored in-depth fathers' experiences during the weeks and months following the death of infants' mothers, including infant hospitalization, legal battles, and the struggle to adjust to life as a widowed father. This study highlights critical needs for support, as well as how those needs are met, or in some cases left unmet.

Results: Four major themes emerged: grief, filling mother's shoes, support, and burden of guilt.

Conclusion: It is imperative that healthcare practitioners treating women during pregnancy make every effort to include fathers throughout the pregnancy, educate fathers as to the mothers' high-risk health concerns, and be prepared to provide local sources of support in the event of maternal death. Practitioners should also be aware that support needs continue to be high throughout the infant's first year of life. Extended family and friends may be involved in the infant's healthcare to help fill the maternal role. 


\title{
2.3.A. Comparison of a Home-Based Motor Development Intervention on Infant Development and Maternal-Infant Bonding Between Late Preterm-Low Birth Weight Infants and Mothers With and Without a Positive Screen of Symptoms of PTSD and/or Depression
}

\author{
Cheryl Anderson, PhD, MS, BSN, CNS, RN; Efret Ghimazion \\ University of Texas at Arlington, Arlington, TX
}

Background: Between $28 \%-43 \%$ of mothers with preterm infants admitted into neonatal intensive care units (NICUs) can experience acute stress symptoms and/or depression; a few may suffer from posttraumatic stress disorder (PTSD). Poor maternal mental health affects mother and infant, supporting the need for early assessment and intervention; yet, few interventions have been examined.

Methods: This institutional review board-approved, longitudinal, pilot study used convenience sampling to recruit 40 women from the NICU for a phase 1 study to collect initial information related to mental health and maternal-infant bonding. For phase 2, by incorporating a randomized block design, phase 1 study women, subgrouped by symptom severity, will be randomly placed in experimental or comparison groups and reassessed in the home at 6, 9, and 12 months postpartum for PTSD and depression symptoms and maternal-infant bonding. Infants in the experimental group will also receive three sessions of the home intervention within 12 months postpartum and assessments via Bayley Scales of Infant and Toddler Development, the Alberta Infant Motor Scale, and the Affordances in the Home Environment for Motor Development. Our home intervention is an innovative, goal-oriented, intensive motor training program that actively involves parent-infant interaction and includes environmental enrichment strategies. ANOVAs/repeated measures ANOVAs are planned to determine differences between women by demographics, symptom severity, and intervention effects.

Results: Currently 25 women are enrolled in phase 1 and show mild to moderate symptoms of depression (15\%$23 \%$ ) and previously diagnosed PTSD (5\%) without impairment in mother-infant bonding. Typically, women are Latina, age 28, with a mean infant gestational age of 32 weeks. Phase 2 begins in January 2019.

Conclusion: Findings are expected to support best practice for assessment and follow-up of at-risk mothers. We believe this intervention can be beneficial to infant development and mother-infant interaction, hence, an indirect reduction in maternal mental health symptoms.

\subsection{B. Where Is the Patient in Interprofessional Collaborative Practice?}

Louanne Friend, PhD, MN, RN; Richard Friend, MD, FAAFP

University of Alabama, Tuscaloosa, $A L$

Background: The literature suggests that interprofessional collaborative practice (ICP) promotes interdisciplinary collaboration and reduces preconceptions among various groups. What is less clear is how often students actually practice as teams when licensed and if interprofessional education (IPE) supports their ability to effectively provide patient-centered care in healthcare teams. This mixed methods study was designed to measure student feedback regarding the influence of IPE postgraduation. We present findings related to the enduring impact of a prelicensure, co-enrolled, critical care elective for upper level nursing and third- and fourth-year medical students.

Methods: Institutional review board approval was obtained, and informed consents were collected. A total of 40 upper level nursing and 14 third-and fourth-year medical students completed the co-enrolled critical care elective during spring semesters 2016 and 2017 and were invited to participate in follow-up surveys. During the course, students met face to face for 2 hours weekly learning commonly performed procedures in addition to effective conflict resolution techniques. Surveys were administered during the spring semester of 2018 using Facebook. The Assessment of Interprofessional Team Collaboration Scale II and two researcher-generated questions were utilized.

Results: A total of 27 of 54 students completed the survey, yielding a return rate of $50 \%$. Overall scores indicated higher group ratings on partnership and cooperation subscales. Lowest items were related to including patients in goal setting and adjusting care plans. Respondents indicated having no agreed upon process to resolve conflicts. Participants reported the course gave them long-term confidence to work in teams.

Conclusion: ICP is defined as working with patients and their families. The data suggest that teaching prelicensure nurses and physicians how to collaborate has enduring impact. However, educators must also focus on the concept of patients as team leaders. Likewise, additional information related to resolving interprofessional conflict is warranted. 


\title{
3.1. Sequencing of Critical Appraisal and Quality Assessment in Joanna Briggs Institute Qualitative Reviews
}

\author{
Marilyn Macdonald, PhD, RN; Ruth Martin-Misemer, PhD, NP, RN
}

Dalhousie University, Halifax, Nova Scotia

Background: Joanna Briggs Institute (JBI) systematic reviews of qualitative research are conducted according to philosophically informed methodology and methods. A stepwise approach is used to develop reviews that are trustworthy and from which lines of action can be drawn. The quality of the studies as well as the quotes used to support themes are assessed in two distinct steps: critical appraisal and quality assessment. The purpose of this presentation is to engage participants in a dialog regarding the pros and cons of conducting the quality assessment of quotes in conjunction with critical appraisal. This facilitated dialog is intended to advance the conference objective of enhancing quality and trustworthiness associated with project designs.

Methods: Examples of findings from a review conducted by the authors will provide context for a facilitated discussion.

Results: The discussion is intended to generate a scholarly dialog related to the intersection of the steps of critical appraisal and quality assessment. The current ordering of these steps has the potential to result in a less robust data sample for synthesis. This occurred in one of our reviews at the quality assessment stage, resulting in downgrading the confidence of the synthesized findings. If the facilitated discussion supports this claim, this would provide rationale to have the JBI Methodology Committee examine the claim and advise whether changes are warranted.

Conclusion: The conduct of a systematic review is intended to generate findings to guide lines of action. The quality of a review is contingent upon the quality of the included studies. Critical appraisal and quality assessment determine the quality of included studies, and a JBI Methodology Committee review that examines the current sequencing of these steps is advised.

\subsection{The Role of Nursing in Advancing Healthcare Quality}

Kim Sears, PhD, RN; Christina Godfrey, PhD, RN

\section{Queen's University, Kingston, ON}

Background: Advancing the quality of healthcare has evolved over the past several decades. This presentation highlights some of the current global initiatives as well as the benefits of having an interprofessional team approach to healthcare quality that were gained from the preliminary results of an umbrella review answering the following research question, "What is the role of nursing in advancing healthcare quality?" The aim of this presentation is to identify the key role that nurses play as part of the interprofessional team to advance healthcare quality.

Methods: The goal of the umbrella review was to capture the current efforts of nurses advancing quality healthcare through systematic reviews. In this high-level synthesis, 364 reviews resulted from the search strategy. They were screened by title and abstract. Multiple databases were identified and searched by the library scientist. The reviews were screened for those describing nurses' roles in advancing quality healthcare. From there, 14 papers were eligible for full-text screening. Critical appraisal was conducted using the Joanna Briggs Institute critical appraisal tool for systematic reviews.

Results: Preliminary findings demonstrate that nurses are pushing the boundaries of traditional healthcare and advancing their roles in multiple settings to strive for improved care, influencing clinical and professional outcomes. These results align with the current global initiatives in quality improvement. The results will be examined through the Donabedian model.

Conclusion: Nurses are key members, and their contribution is to provide insight into care planning, quality of life and patient satisfaction, prevention strategies, and patient education delivery. Nurses need to lead and contribute to quality advancement, quality assurance, problem resolution, and quality improvement regardless of specialty, role, or qualification. With nurses holding a multifaceted, autonomous, and integrative job, quality improvement needs to be ingrained in the ethical standards guiding practice for patient care to continue to advance. 


\section{P1. Improving Nursing-Sensitive Patient Outcomes Through Shared Governance}

Joseph Eppling, MN, RN, CRRN, NEA-BC

University Medical Center, New Orleans, LA

Background: Healthcare programs from inpatient hospitals to providers are focused on quality more than ever before. Providing quality care to patients is not only the right thing to do, but the reimbursement structure continues to evolve and revolves around patient outcomes with regard to payment for services. Specifically, the Centers for Medicare and Medicaid Services has implemented value-based purchasing that penalizes providers for poor quality care and never events such as hospital-acquired conditions (HAC). Nurses providing direct care to patients in the hospital are in the position to have an impact on reducing or eliminating HACs, thereby improving patient outcomes. Bedside nurses providing direct care need to be empowered to make necessary changes that influence nursing practice and impact patient care. Studies have demonstrated that a shared governance structure provides the work environment for empowering staff nurses as well as improving nursing satisfaction.

Methods: The purpose of this quality improvement project was to create a nursing quality council (NQC) in a shared governance environment. The NQC was established with frontline staff nurses. The overall goal is to empower frontline staff nurses with the ability to impact the work environment that will lead to improved quality and care for patients. The specific quality indicators will focus on nursing-sensitive patient outcomes including catheter-associated urinary tract infections (CAUTI), central line-associated bloodstream infections (CLABSI), falls, and hospital-acquired pressure ulcers (HAPU).

Results: While a two-sample z-test allowing unequal variance did not prove to be statistically significant, the preliminary results demonstrate an improvement in falls and HAPU, with 2 of the 4 nursing-sensitive patient outcomes being measured.

Conclusion: This scholarly project was successful and met the aims and objectives. One of the "five must haves" for shared governance, a quality council, was established involving frontline, direct care staff nurses.

\section{P2. Creation of an Interprofessional Evidence-Based Practice Council for Collaborative Practice}

Donna Copeland, DNP, RN NE-BC, CPN, CPON, AE-C; Kristina Miller, DNP, RN, PCNS-BC, CNE

University of South Alabama, Mobile, AL

Background: An inundation of evidence, lack of confidence in translating evidence into practice, and a deficiency in working in interdisciplinary teams hinders healthcare providers from delivering the highest quality of care. To narrow the gap between knowledge generation and knowledge translation, providers from all disciplines must be able to communicate and collaborate with one another effectively. Therefore, a strategy for empowering interprofessional teams in the process of implementing evidence-based practices (EBP) to improve patient care is paramount. The purpose of this project was to develop an Interprofessional Evidence-Based Practice Council (IEBPC) to engage frontline staff in research, implementation of EBP, and interprofessional-shared governance to accelerate change within a hospital setting.

Methods: Through an academic-practice partnership, a steering committee was formed, and hospital administrative support was obtained. Before guiding staff through the process of EBP and working in interprofessional teams, baseline data were obtained. Four instruments were utilized to gain insight into the current state of staffs' beliefs and competence, frequency of knowledge translation, and perceptions of the organizational culture and readiness for integration of EBP: EBP Beliefs Scale, EBP Implementation Scale, EBP Competence Scale, and Organizational Culture and Readiness for System-Wide Integration of EBP Scale.

Results: Findings indicated that although staff believe that EBP is effective in improving clinical care, there is a gap in the staff's understanding of fundamental concepts of EBP, the processes necessary to find and translate evidence into practice, and a lack of formalized training on working in interprofessional teams. Overall, staff reported potential satisfaction in participating in an IEBPC and were engaged in the process of improving the organizational readiness for the integration of EBP. These results will be used to guide further improvements in the development of the council.

Conclusion: Engaged frontline staff champions and leaders have the opportunity to play an integral role in the development of EBP cultures. This project is ongoing; however, it is hypothesized that the implementation of an IEBPC that utilizes an interprofessional shared governance model, as well as training in EBP and interprofessional collaboration, will have far-reaching patient-centered outcomes. 
P3. Partners in PIVIE Prevention: Engaging Leadership, Team Members, and Families in Reduction of Harm

Angela Seput, BSN, RN; Kathleen Vincent, RN

Children's Hospital, New Orleans, LA

Background: Historically, a medical/surgical unit with high patient volume and patient turnover experienced a high rate of peripheral intravenous infiltration and extravasation (PIVIE) (In 2015, the total PIVIE rate was 13.65 per 1000 patient days). In 2015, the unit began active participation in the hospital-wide PIVIE team which included the implementation of a prevention bundle. Following a spike in the rate in early 2017, unit leadership began to engage in intensive PIVIE reduction efforts.

Methods: Quality improvement unit leadership, using lean rapid process improvement principles, began leadership rounding every weekday on patients with peripheral intravenous lines (PIVs) starting in April 2017. Rounding included examining the PIV site, ensuring the "Touch-Look-Compare" (TLC) poster was actively utilized during each assessment, and educating and engaging family members in the TLC process. Missed bundle elements were resolved daily and data visually displayed in posted Pareto charts and the Kaizen Newspaper for ongoing improvement. In September 2017, night-shift team members were added to the PIVIE team. Team engagement efforts included encouragement to report mild PIVIEs and engaging child care technicians to examine PIV sites and to complete TLC assessment when vital signs are obtained.

Results: From January 2016-April 2017, the unit's total PIVIE rate was 7.90 per 1000 patient days. From May 2017February 2018, the rate was 4.67 per 1000 patient days $(P$ value $=0.021$; utilizing a two-proportion test). The rate reduction was sustained after leadership transitioned daily rounding to clinical leaders.

Conclusion: An intensive leadership rounding process led to a sustained drop in PIVIE occurrence. Next steps include ongoing education and training for this process to other medical/surgical units. Additionally, the leadership teams will continue to engage new team members in PIVIE prevention to ensure sustained results.

\section{P4. Improving Communications and Perceptions of Mutual Support to Expedite a Major Perinatal Practice Change}

Lori Noel, MSN, BSN, RN, CNOR

Ochsner Medical Center, Kenner, LA

Background: Increased newborn patient falls and adverse respiratory events identified failures in transitioning care from delivery to the first 2 hours after birth. Transition care models were not finalized, and best practice recommendations were loosely enforced. Perinatal staff were administered the TeamSTEPPS Teamwork Attitudes Questionnaire (T-TAQ) which identified poor communications and lack of team support. Through an interprofessional collaborative committee (ICC), this project sought to improve mutual support perceptions and team communications to implement practice change in a change-resistant environment.

Methods: Evidence-based research was applied to create a perinatal ICC that included representatives of all shifts and units, champions and detractors, and unit leaders. TeamSTEPPS Mutual Support education and simulation training was administered to nursing and ancillary staff. A newborn transition-in-care operational standard was created, and staffing practices were revised. The new model, based on the Association of Women's Health, Obstetric and Neonatal Nurses' guidelines, required the labor nurse to remain at the bedside during the 2-hour transition period. Complexity theory was applied, and Plan-Do-Study-Act (PDSA) and debriefing tools were utilized during model implementation.

Results: T-TAQ results were unanimous with all 21 survey respondents choosing "disagree" or "strongly disagree" to the statement "personal conflicts between team members do not affect patient safety." The ICC identified strategies, determined data collection and evaluation methods, and outlined responsibilities. Policy and operational standards were completed, the business case was presented to organizational leadership, and the new staffing model was initiated. PDSA and debriefing tools have shown minimal staff concerns and difficulty in implementation.

Conclusion: Results of the T-TAQ demonstrated the integral relation between patient safety and work environment. By focusing on the staff's unanimous response and addressing that issue directly, we were able to rapidly implement a new care model in a change-resistant environment. 
P6. Integrating Palliative Care and Heart Failure Services

\author{
Lauren R. Ellicott, DNP, RN-BC, ACCNS-AG
}

Mount Carmel Health System, Columbus, $\mathrm{OH}$

Background: A large volume of patients with heart failure go without the support of palliative care services. It is uncommon for providers to initiate palliative care referrals for patients with heart failure. Currently, there are gaps in the literature with respect to integrating palliative care services with patients who have heart failure. The purpose of this patient quality improvement project was to increase palliative care and heart failure integration knowledge and awareness for healthcare providers who treat patients with heart failure.

Methods: A quality review was conducted to observe the impact of heart failure palliative care integration education on the amount of palliative care service referrals initiated for heart failure patients. Five advanced practice nurses working in an outpatient heart failure clinic were recruited and asked to complete an online palliative care heart failure management educational course and implement what they learned into their everyday practice. Pre- and posteducational counts of palliative care referral initiation were collected for data analysis.

Results: Descriptive statistics were used to summarize the number of palliative care referrals made by the advanced practice nurses one month before and one month after the palliative care heart failure education. A total of 578 heart failure patients were seen. Zero of 290 patients were referred to palliative care services during the pre-education month, and only one of 288 patients received a palliative care referral during the post-educational month.

Conclusion: Provider integration of palliative care and heart failure services does not improve with an online educational course alone. More time, focus, and attention to knowledge deficits need to be directed towards enhancing healthcare provider insight of integrating heart failure and palliative care management.

\title{
P7. Replicating a Fall Prevention Program in an Outpatient Endoscopy Unit
}

Escel Stanghellini, DNP (c), MSN, RN, CPHQ

Brigham and Women's Hospital, Boston, MA

Background: Falls are among the top causes of mortality in older adults. An estimated 2.8 million older adults in the United States are evaluated in emergency rooms for fall-related injuries. The national expenditure to treat fall-related injuries is expected to increase to $\$ 67.7$ billion by 2020 . Falls are recognized as an indicator of the quality of nursing care. Procedures that previously required inpatient hospitalizations are now performed in outpatient settings where patients are also at risk for falls. Fall prevention processes used in inpatient settings can also be used in outpatient settings. The purpose of this project was to assess the impact of implementing an evidence-based fall prevention program that included fall risk assessment, tailored interventions, and patient education to reduce the number of falls and injuries from falls in an outpatient endoscopy unit.

Methods: The project took place in an outpatient endoscopy unit in a large academic center in Boston. Support from the endoscopy unit leadership and nurses was obtained. Nurses were trained during a 2-week period in January 2018. Process data (patient awareness of fall risk and plan) and outcome measures data (falls) were collected in December 2017 (pre) and in March 2018 (post). The Donabedian structure, process and outcome model and the Plan-Do-Study-Act cycle for rapid cycle improvement were used.

Results: Fall rate did not change given the low rate of falls and the limited time to complete the project. However, the increase in patient awareness showed statistically significant improvement $(P=0.05)$, resulting in improved care processes. This suggest a potential for improved outcomes with further examination.

Conclusion: Training can result in competence, leading to improved quality in the fall prevention process. Expanding the duration of the project is necessary to determine if outcomes can be replicated in other outpatient settings. 
P8. The Association of Premorbid Neuropsychological Conditions With Pediatric Mild Traumatic Brain Injury/Concussion Recovery Time and Symptom Severity: Systematic Review Findings

\author{
Michelle Goreth, DNP, APRN, CPNP-AC, CTRN, CCRN, CPEN, TCRN \\ University of Mississippi Medical Center, Jackson, MS
}

Background: Pediatric mild traumatic brain injury, or concussion, is an increasing population health concern. Some studies suggest that children with preexisting neuropsychological disorders such as behavioral disorders, learning disabilities, or mood disorders have a longer duration or increased symptoms after mild traumatic brain injury compared to children without premorbid conditions, but the evidence is mixed. Little scientific inquiry has been conducted to identify the impacts of concussion in children with premorbid neuropsychological conditions (PNPC), as they are often excluded from research because of their PNPC.

Methods: A systematic review utilizing the Joanna Briggs Institute (JBI) methodology for reviews of etiology and risk was conducted with an a priori protocol to identify the associations of premorbid neuropsychological conditions on mild traumatic brain injury/concussion recovery time and symptom severity in children.

Results: Analysis revealed 12 articles for systematic review inclusion with significant study heterogeneity, as well as variation in study findings. Thus, a metaanalysis was unable to be conducted, and a JBI Grade of Evidence B was applied to findings.

Conclusion: Significant associations for prolonged recovery or increased symptom severity after mild traumatic brain injury were identified in children with premorbid histories of learning disorders and poor academic achievement; anxiety, depression, mood disorders, or other psychiatric illnesses; prior head injuries (especially with a history of multiple concussions); somatization (in females); sleep disorders (in males); and multiple neuropsychological conditions. Significant research gaps exist, but overall findings suggest that clinicians should incorporate postconcussion screening for premorbid neuropsychological conditions and closely monitor injury recovery.

\title{
P9. Using Critical Incident Stress Debriefing to Impact Perception of Support During Crisis in Adult Inpatient Nurses
}

Shannon West, BSN, RN, PCCN, CCRN-K

Ochsner Medical Center, New Orleans, LA

Background: Exposure to repeated stressors places healthcare workers at risk for compassion fatigue and burnout. Debriefing mitigates crisis and contributes to feelings of mutual support. A survey of registered nurses (RN) at a large, academic healthcare facility showed their perception of support during crisis varied from no support to excellent support, and $86 \%$ rated support as very important/essential. In 2017 , a multidisciplinary team implemented the code lavender pilot for RNs on units with high mortality rates, aiming to determine the effectiveness of critical incident stress debriefing (CISD) and structured support on RNs' perception of workplace support during crisis.

Methods: Chaplains and patient flow coordinators (PFC) received training using the CISD tool to serve as responders who debrief nurses in crisis. Nurses received information on when and how to activate code lavender for individuals or groups experiencing crisis. Upon activation of code lavender, the response team, consisting of a chaplain, PFC, and nursing administrator, responded to the unit. Effectiveness of code lavender was evaluated through a postintervention survey of nurses and responders.

Results: Three months after initiating the pilot, code lavender was activated 4 times for individuals and once for a unit. The postintervention survey revealed that nurses appreciated the support but felt averse to responders due to the perception that responders did not understand the nurses' experience. The survey indicated that RNs did not activate code lavender on multiple occasions when criteria were met due to the perception that the response was not helpful. Feeling of support during crisis was not met due to lack of program utilization. The survey showed that chaplains and PFCs felt the need for additional training.

Conclusion: Barriers to utilization of code lavender were lack of buy-in and poor perception of response. We plan to provide formal training for responders to provide more robust support for RNs experiencing crisis in the future. 
P10. Older Nurses' Experiences of Providing Direct Care in Hospital Nursing Units: A Qualitative Systematic Review

\author{
Alice Gaudine, PhD, RN; Karen Parsons, PhD, RN \\ Memorial University of Newfoundland, St. John's, Newfoundland
}

Background: Most developed countries are experiencing an aging workforce as their population ages. The retirement of aging nurses has contributed to a critical global nursing shortage that is anticipated to increase. This global nursing shortage is partly due to a consistent pattern of nurses leaving hospital settings or the workforce after the age of 50 years. The many reasons why older nurses leave the workforce early include shift work, the stress of a heavy workload, and perceived lack of responsiveness to nurses' concerns and chronic health conditions. It is important to understand the experience of older nurses in high-paced hospital nursing units. This systematic review integrated the literature on the experiences of older nurses providing direct nursing care.

Methods: This review considered studies that included registered nurses $\geq 45$ years who work as direct care nurses on any type of inpatient hospital nursing unit. This review considered studies that focused on qualitative data including the following methodologies: phenomenology, grounded theory, ethnography, action research, and feminist research. The databases searched included: CINAHL, PubMed, Psycinfo, Embase, AgeLine, Sociological Abstracts, and SocINDEX; the search was conducted on October 13, 2017.

Results: Three synthesized findings were identified consisting of 12 categories and 75 findings. The 3 synthesized findings extracted from the papers were (1) the love of nursing: it's who I am and I love it; (2) it's a rewarding but challenging and changing job; (3) it's a challenging job: can I keep up? Implications for nurse leaders and healthcare organizations stemming from our findings are identified.

Conclusion: Older nurses love nursing and have created an identity around their profession, yet they identify many challenges and changes. To address the workforce shortage, it is important to address the implications of our findings for nurse leaders and healthcare organizations.

P11. Certification Central: Increasing Critical Care Registered Nurse Certification in the Pediatric Intensive Care Unit

Jamie A. Harrell, RN, BSN; Angela Layden, RN, CCRN

Ochsner Medical Center, New Orleans, LA

Background: Critical care nursing certification (CCRN) is a valued component in the education and confidence of a registered nurse. By obtaining the certification, the nurse not only develops and deepens his or her own education and understanding of nursing practice but also puts the patient's safety and care at the top of the priority list. It allows for the nurse to care for both the patient and the family, as well as contribute to the pediatric intensive care unit (PICU) team. Certification allows the nurse to demonstrate how to care for critically ill patients and show proficiencies in knowledge and skill involved in the critical care area. Louisiana had a 16\% certification rate as of 2017 . The PICU has a $32.9 \%$ certification rate. The intent of this project was to bring awareness to the importance and value of CCRN certification on both the personal and professional level.

Methods: A unit ambassador was assigned to speak in groups as well as individually to interested staff nurses. Materials were included in the new hire packets for future test taking, and resources were provided for study materials. The ambassador recently took the certification examination herself and ensures that individuals know where to sign up and find the appropriate materials to study for the examination. The ambassador also works to promote a culture of certification among the unit. The ambassador focuses on personal and professional growth, the benefits to Ochsner of obtaining certification, and the need for continued participation on the unit.

Results: The intent of this project was to increase the number of staff RNs considering and completing CCRN certification. Our initial percentage was $32.9 \%$, and the goal was $45 \%$ over the next 12 months. After 5 months of implementing this practice, $37.5 \%$ of the staff qualified to sit for the exam, a $4.5 \%$ increase. The unit has had 2 additional nurses take the certification and pass. Three additional nurses expressed interest and/or have signed up for the test.

Conclusion: With the help of the ambassador and the encouragement of other certified nurses, there is great potential to increase the number of CCRN-certified PICU nurses to ensure improved quality of care and safety to PICU patients. The certification percentage has increased with those eligible to sit for the test. All new hires have been presented with materials for the certification and will be contacted once they meet the requirements for taking the test. 
P12. Implementation of an Educational Session and Simulated Clinical Experience on Responding to Code Blue Emergencies: A Quality Improvement Project

\author{
Brandy Barbarin, MN, BSN, RN \\ LSU Health School of Nursing, New Orleans, LA
}

Background: The American Heart Association (AHA) established the Get With The Guidelines - Resuscitation (GWTG-R) quality improvement program to optimize outcomes through benchmarking, knowledge translation, and research. Three of the metrics tracked by GWTG-R are (1) initiate cardiopulmonary resuscitation (CPR) within 1 minute of cardiac arrest, (2) defibrillate within 2 minutes for shockable rhythms, and (3) administer epinephrine within 5 minutes for asystole and pulseless electrical activity. Baseline data on these 3 metrics were obtained from a large, teaching tertiary hospital located in an urban community in the Southeastern Region of the United States. Initiating CPR within 1 minute, defibrillating within 2 minutes, and administering epinephrine within 5 minutes in at least $85 \%$ of in-hospital cardiac arrests were not consistently being met. Studies show simulation training has a positive effect on staff performance and improved patient outcomes in code blue emergencies.

Methods: For this project, a doctor of nursing practice student conducted educational sessions with code blue simulation and debriefing with registered nurses and patient care technicians on an inpatient medical-surgical unit for a period of 4 weeks. The sessions focused on collecting data during the first 5 minutes of the simulated cardiac arrest. The postsimulation debriefing topics of discussion included communication issues, safety, staff confidence, and compliance with AHA guidelines for in-hospital cardiac arrest response. The aims of this project were to improve code blue performance, patient outcomes, and resource utilization.

Results: All participants were basic life support-certified by the AHA. Data on time to initiating CPR and time to first shock was collected utilizing the code blue audit tool. The mean time to initiate chest compressions during the simulation sessions was 20.8 seconds $(n=12)$. The mean time to deliver the first shock during the simulation sessions was 78.8 seconds $(n=12)$. The results of the simulation effectiveness tool indicate that $100 \%$ of the participants $(n=36)$ found the simulation sessions prepared them to respond to changes in patients' conditions and increased their confidence in their ability to prioritize care and interventions, communicate with patients, teach patients about their illness, report information to the healthcare team, foster patient safety, and use evidence-based practice to provide nursing care.

Conclusion: Overall, the simulated clinical experience had a significant positive effect on performance in a code blue situation.

P13. Correlation of Primary Learner Assessment and Electronic Fetal Monitoring Education on RN Learning Outcomes

Sharon Cusanza, MSN, RN, NEA-BC

LAMMICO/Medical Interactive, Metairie, LA

Background: Timely and accurate interpretation of electronic fetal monitoring (EFM) data is critical to the safety of both a mother and fetus. To promote patient safety and mitigate risks related to this high-risk skill, Medical Interactive (MI) offers unique computer-based education through the Learning Management System (LMS). Upon completion of an EFM Primary Learner Assessment (PLA), the LMS generates an individualized learning plan of continuing education course recommendations to meet identified knowledge gaps. From an evidence-based practice perspective, research is warranted to determine improvements in knowledge and skills as well as patient safety perceptions as a result of completing these courses through the PLA in the LMS. Therefore, the primary purpose of this research study was to quantify changes in RN knowledge and skills as measured by improvement in preassessment and postassessment scores for the PLA obtained from the participant assessment and survey.

Methods: This survey research study included 55 RNs who work in labor and delivery units administering and interpreting EFM from 3 hospitals. Participants completed surveys and recommended EFM-related education. Subject duration was approximately 1 month for completion of the initial survey, continuing nursing education (CNE) courses, and associated pretest/posttest; then, a follow-up survey was completed approximately 15 days thereafter.

Results: The average RN knowledge and skills between preassessment (73.3\%) and postassessment (85.2\%) scores showed a statistically significant improvement (paired $t$-test $=7.96, P(\mathrm{~T} \Leftarrow \mathrm{t})$ two-tail $<0.0001$ ). There were also improvements between aggregated averages of pretest $(70.1 \%$ correct) and posttest $(80.5 \%$ correct) scores from completion of all the recommended and highly recommended CNE courses. An improvement was noted in averaged EFM practice-related questions obtained from the participant surveys at baseline $(44 \%)$ and follow-up $(93 \%)$ as well.

Conclusion: As a result of the PLA and completion of recommended EFM courses through the learner-directed assessment and targeted education, the RN participants' knowledge and skills were improved. RN perceptions regarding outcomes were improved oxygen therapy, competence, and interpreting fetal rhythms and resulting interventions. 
P14. Parent Satisfaction With Feeding Competence in Preterm Infants: Infant-Driven Feeding Compared With Standard Scheduled Feeding

Carrie Mayo, BSN, RN

Louisiana State University Health New Orleans, School of Nursing, New Orleans, LA

Background: Providing correct education about infant-driven feeding (IDF) while the infant and family are in the hospital can prevent the need for long-term rehabilitation after discharge. There is limited research on parent satisfaction with feeding education, especially comparing standard scheduled feeding (SSF) education with IDF. This project evaluated a feeding policy change from SSF to IDF and the resulting parent satisfaction with comfort of feeding at discharge.

Methods: Parents were interviewed and surveyed prior to discharge regarding education and feelings of competence with feeding. After discharge, parents received the standard discharge call. All were asked about feeding progress and weight gain after discharge. A retrospective review of discharge surveys compared parent satisfaction and education with infants fed using SSF. The goal was to survey $50 \%$ or more of eligible parents during the 12-week implementation phase, with $100 \%$ of parents on IDF receiving education, and $85 \%$ of infants gaining weight between discharge and the first pediatrician appointment.

Results: Seventy-two percent of eligible parents participated in the evaluation, $100 \%$ of the parents surveyed were taught how to feed infants by reading cues, and $84 \%$ felt very confident or confident in their ability to feed at discharge. More than $95 \%$ of parents stated an understanding of reading an infant's readiness cues before and during feeding at discharge. More than $95 \%$ of infants gained weight between discharge and the first pediatrician visit.

Conclusion: Parents of infants discharged on IDF feedings felt more confident at home than the parents of those discharged on SSF. IDF required greater parent education from nursing staff and therapists; however, evaluation demonstrated increased parent satisfaction, greater confidence at discharge and athome, and positive infant weight gain.

P16. Getting to Zero: Eliminating Unplanned Extubations in the Pediatric Intensive Care Unit

Anne Pirrone, BSN, RN, CCRN; Chelsea Mahoney, BSN, RN

Ochsner Hospital for Children, New Orleans, LA

Background: Unplanned extubation (UE) is a serious adverse event that has the potential to cause severe hypoxia, increased risk of infection, airway trauma, prolonged mechanical ventilation, and increased length of stay. Additionally, UE is associated with an increase in costs due to increased supply usage, additional imaging and laboratory studies, and increased staffing requirements. Identified risk factors for UE include younger age, agitation/delirium, inadequate tube fixation, weaning from mechanical ventilation, bedside procedures, and high patient-nurse ratio. The published rates of UE in the pediatric population range between 0.11-6.4 events/100 ventilator days. The purpose of this project was to decrease UE in the pediatric intensive care unit (PICU) to $<1$ occurrence per 100 ventilator days using evidencebased strategies.

Methods: Using Plan-Do Study Act methodology, the following cycles for change were implemented over a 2-year timeline: education on proper sizing and application of commercial tube holder and root cause analysis of each UE that revealed 2 commonalities (undersedation and improper securement) that were consistent with published literature. Additional interventions included leader bedside rounding on the security of the endotracheal tube, implementation of a sedation-titration protocol to give bedside nurses greater autonomy in determining treatment for agitation, and standardization of the method to secure endotracheal tubes. Retrospective data was used to track the UE rates, preimplementation and postimplementation of these evidence-based interventions.

Results: Since project inception, the UE rate decreased from 1.4/100 ventilator days to $0.3 / 100$ ventilator days with the implementation of evidence-based strategies. As a result of this quality improvement project, the PICU went 22 months without a UE event.

Conclusion: Through the implementation of an interprofessional quality improvement program, it is possible to reduce the incidence of UE adverse events. Ongoing monitoring and analysis will be essential to maintaining these low UE rates. 
P17. The Association Between Maternal Exposure to Particulate Matter 2.5 and Neonatal Diagnosis of Congenital Heart Defects: A Systematic Review

Katie C. Hall, MSN, RN, CCRN-K; Jennifer Robinson, PhD, RN, CNE, FAHA

University of Mississippi Medical Center, Jackson, MS

Background: Air pollution is a major environmental risk factor impacting individuals' health with links to asthma, heart disease, obesity, and fetal developmental complications. Particulate matter 2.5 (PM2.5) is one air pollutant with such associations to one's health, especially among vulnerable populations. The development of a congenital heart defect (CHD) is a fetal complication that impacts 34.3 million infants globally with $>80 \%$ of the diagnoses having an unknown etiology. Although environmental factors such as air pollution are thought to be a risk factor in the diagnosis of a CHD, epidemiologic research evidence is limited. Therefore, the purpose of this review was to determine if there is an association between maternal exposure to PM2.5 during the first trimester of pregnancy and a CHD diagnosis.

Methods: The Joanna Briggs Institute quantitative systematic review method for search strategies, critical appraisal, and quantitative data extraction was followed. Findings were reported in narrative format and as metaanalysis when statistical pooling of the data was possible.

Results: Five of 2,295 studies were identified as meeting the inclusion criteria. Each individual study identified at least one statistically significant increase or inverse association between PM2.5 and a CHD. Metaanalysis results were conducted on 3 isolated CHDs identified in more than one study: atrial septal defect (odds ratio [OR] $=0.65$; 95\% confidence interval [Cl] 0.37-1.15); ventricular septal defect ( $\mathrm{OR}=1.02 ; 95 \% \mathrm{Cl} 075-1.37$ ); and tetralogy of Fallot $(\mathrm{OR}=1.16 ; 95 \% \mathrm{Cl}$ 0.78-1.73).

Conclusion: In our analysis, no significant evidence supported an association between pollutant PM2.5 and a CHD in the first year of life. However, few studies met the rigorous inclusion criteria, and the studies that did had high heterogeneity, making it difficult to complete a metaanalysis on such a limited number. Further research is needed to standardize the outcomes, standardize pollutant monitoring methods, and provide comparable analysis results for future synthesis of the literature.

P18. Effectiveness of Interstage Home Monitoring on Survival Rates, Weight Gain, Hospital Admissions, and Cost Savings in Infants With Hypoplastic Left Heart Syndrome: A Systematic Review

\author{
Chelsey Andries, DNP, RN, CPN; Michelle Palokas, DNP, RN-CPN \\ University of Mississippi Medical Center, School of Nursing, Jackson, MS
}

Introduction: Infant mortality rates linked to hypoplastic left heart syndrome (HLHS) have declined since the discovery of 3-stage surgical palliation but remain high between the first and second stages due to the delicate state of the infant. Along with high mortality rates, infants in the interstage period tend to have higher hospital readmission rates, poor weight gain, and longer hospital lengths of stay, all of which result in higher costs. Healthcare institutions must strategically plan to manage these patients to reduce frequent hospital readmissions and reduce costs while improving the healthcare outcomes of these infants.

Methods: The Joanna Briggs Institute method of synthesis was used to perform the systematic review. The steps performed to complete the review included developing a protocol, stating a question, identifying inclusion criteria, detailing a search strategy, critically appraising the identified studies, extracting data, and synthesizing and summarizing the findings.

Results: The 4 studies included were pooled for a metaanalysis and demonstrated statistical significance for the utilization of interstage home monitoring on reducing the odds of mortality rates by $85 \%$. The variance in the reported results for interstage weight gain did not allow for statistical pooling; therefore, these results were presented as a narrative synthesis. The 2 studies that reported weight gain did not demonstrate a significant difference with the utilization of home monitoring. None of the studies used in the review reported on hospital admissions or cost savings related to interstage home monitoring.

Conclusion: This review provides clear evidence of the significance of implementing interstage home monitoring into practice as a standard of care for infants with HLHS. Interstage home monitoring improves survival and helps monitor weight gain trends of infants with HLHS. Home monitoring is a noninvasive intervention with little to no adverse effects. Health professionals should utilize home monitoring during the interstage period to improve health outcomes in patients with HLHS. 
P19. Clinician Knowledge of Perinatal Mood and Anxiety Disorders in Florida

Margaret Salib-Barrios, DNP, APRN, FNP-c; Susan Liipfert Shelton, PhD, ARNP, CNM

Florida State University, Tallahassee, FL

Background: The purpose of this project was to understand knowledge gaps and barriers among Florida healthcare providers in the assessment and treatment of perinatal mood and anxiety disorders (PMADs). The information gathered will assist in making future recommendations to improve early screening and identification of women with PMADs.

Methods: The study was designed as a needs assessment questionnaire. The study was approved by the Florida State University (FSU) human subjects committee prior to initiation. Advanced registered nurse practitioners physician assistants, doctors of osteopathic medicine, and medical doctors (MDs) with email addresses listed in the Florida Health Care Practitioner Data Portal were sent an investigator-developed survey. Upon completion, participants were provided a link to MothersMentalHealth.org, developed by the FSU College of Medicine, that provides resources and education for mothers, families, and clinicians. Analysis was conducted using the Statistical Package for the Social Sciences to determine how clinicians identified areas where they lacked preparedness in addressing PMADs and to identify differences in provider characteristics.

Results: A total of 552 respondents completed the survey. The top 6 areas clinicians lack preparedness included appropriate use of language, detection of PMADs, care development, time and resource management, awareness of community resources, and provision of culturally specific care. Significant differences in preparedness were observed between disciplines but not in provider sex.

Conclusion: Barriers to effectively addressing PMADs are all modifiable. Efforts are needed to improve the confidence, knowledge, and preparedness of all clinicians, regardless of specialty area, in the early detection, appropriate treatment, and long-term management of PMADs. Successful management of PMADs is critical for the welfare of women and their families. To further this research, it would be valuable to have PMAD education built into the requirements for all healthcare providers across the state of Florida. Simply increasing the awareness of available resources could improve PMAD outcomes.

P20. Navigating a Spider's Web: Challenges in Qualitative Systematic Reviews

Karen Parsons, PhD, RN; Alice Gaudine, PhD, RN

Memorial University of Newfoundland, School of Nursing, St. John's, Newfoundland

Background: In conducting 2 qualitative systematic reviews ("Older Nurses' Experiences of Providing Direct Care in Hospital Nursing Units: A Qualitative Systematic Review" and "The Experience of Accessing Specialized Healthcare Services for Older Persons Living in Rural or Remote Areas: A Systematic Review Protocol of Qualitative Evidence"), we faced a number of decision points that we believe challenge many qualitative systematic reviewers. Numerous decision points are not as clear-cut as a novice systematic reviewer might assume.

Methods: While conducting our 2 systematic reviews, we kept notes that helped us fully describe the decisionmaking process at each decision-making point we encountered. These will provide clear examples of our quandaries and our decisions.

Results: We identified challenges in conducting the critical appraisal checklist for the inclusion of manuscripts. We also identified issues with data extraction. Some of these issues are related to different approaches to known qualitative methodologies. We identify how the review would have progressed if another direction had been taken and the final impact on the overall findings.

Conclusion: We discuss the value of refinement of guidelines for qualitative systematic reviews to provide consistency in how novice and experienced reviewers conduct qualitative systematic reviews. This will ultimately provide these reviews with more rigor. We will also relate our challenges to those discussed in the literature. The issues we identified, along with the decisions we made, are the types of examples that need to be included in the Joanna Briggs Institute systematic review methodology and in training programs. 
P21. Bathing High-Risk Neonates: Implementation of a Clinical Practice Guideline

Melissa G. Morse, BSN, RNC

LSU Health School of Nursing, New Orleans, LA

Background: The frequencies and techniques of neonatal bathing are well established in evidence-based practice. Various hospitals and professional organizations have transmitted such evidence into clinical practice guidelines, with the purpose of generating best-practice implementation by healthcare professionals to provide safe and quality patient care. However, a wide variation of non-evidence-based neonatal bathing practices remains. The purpose of this project was to implement the bathing practice recommendations in the Association of Women's Health, Obstetric and Neonatal Nurses 2018 publication Neonatal Skin Care: Evidence-Based Clinical Practice Guideline. The project's aims included educating $100 \%$ of the nursing staff and achievement of bimonthly incremental compliance increases over 6 weeks, with $100 \%$ compliance upon project completion. The sustainability goal was incorporation of the bathing clinical practice guideline in the unit's Routines and Guidelines for Care in the NICU handbook.

Methods: The Registered Nurses Association of Ontario Knowledge to Action (KTA) framework was used to guide the project. After collecting baseline bathing data, the nursing staff was intensely educated on the clinical practice guideline, and compliance with the practice change was measured through direct observation and an implementation participation questionnaire.

Results: Compliance with the bathing clinical practice guideline increased from $82 \%$, to $91.5 \%$, and to $97 \%$, and most significantly, daily bathing rates were reduced from $93.6 \%$ to $0.01 \%$. There was positive improvement in all measured preimplementation techniques toward desired postimplementation techniques. Clinical practice guideline noncompliance was usually due to patient criticalness/instability or parental preference.

Conclusion: The clinical practice guideline was successfully implemented using the education-focused KTA framework. Management support of and involvement in the culture change, provision of evidence to support practice recommendation changes, and ongoing project champion availability were key to maintaining clinical practice guideline acceptance, momentum, and sustainability.

P22. Reducing Unplanned Extubations Through Unit-Specific Interventions

Rosanne Hallford, BSN, RN, CCRN; Garrett Lambert, BS, RRT-NPS

Children's Hospital New Orleans, New Orleans, LA

Background: Children's Hospital New Orleans joined a collaborative aimed at reducing unplanned extubations (UE) in January 2016. After 1 year of data collection and analysis, the UE team determined that each intensive care unit (ICU) had different UE etiologies and therefore required individualized interventions to reduce UE rates.

Methods: The UE team reviewed 2016 events to identify trends in unit-specific etiologies. Loose tape was the primary cause of UE events in the neonatal ICU (NICU). Inadequate restraints and inadequate sedation leading to agitation were the causes of UE events in the cardiac ICU (CICU). Through collaboration with the UE team, ICU leaders developed interventions using Plan-Do-Study-Act methodology to target the UE events. In March 2017, the CICU began an intensivist-led rounding process that focused on early identification of patients ready for extubation. In August 2017, the NICU developed an airway card that enabled frontline staff and providers to identify the appropriate location for endotracheal tube (ETT) securement. Use of the airway card allowed for early identification of ETT migration and an opportunity for corrective action prior to UE.

Results: Following the interventions, the CICU experienced a decrease in ventilator utilization (ventilator days divided by patient census days). Ventilator utilization was 0.48 prior to the start of intensivist-led rounding in March 2017. Postintervention, the utilization was 0.33 ( $P=0.000$, two-proportion test). The NICU experienced a decrease in UE rate after implementation of airway cards. Prior to the intervention, UE rate was 0.816 per 100 ventilator days. Postintervention, UE rate was 0.368 ( $P=0.038$, two-proportion test).

Conclusion: Adopting unit-specific interventions has resulted in sustained reductions in the NICU UE rate and sustained reduction in ventilator utilization in the CICU. Next steps include implementation of multidisciplinary rounding and readiness to extubate in all ICUs. 
P23. Catching Up With the Real World of Clinical Support for Breastfeeding Mothers: Changing Demographics, Associated Risk Factors, and Proactive Interventions

Jean Rucks Rhodes, PhD, CNM, IBCLC

Rhodes Research \& Consulting, LLC, Mt. Pleasant, NC (DISCLOSURE: Author is consultant for Medela.)

Background: Suboptimal breastfeeding continues to be a major public health challenge despite recent intense efforts to promote and support breastfeeding in the early postpartum period. While new evidence identifies several prominent physical and demographic risk factors that impede timely milk production-including older maternal age, first-time mothers, increased maternal body mass index, and mode of delivery-little has been done clinically to recognize and address these growing concerns.

Methods: A review of relevant literature identifies multiple maternal risk factors for delayed and/or suboptimal milk production as well as their proposed physiologic basis. Additionally, research to support pump-dependent mothers suggests short-term proactive practices that promote initial and long-term breastmilk production in breastfeeding women.

Results: Recommendations discussed in this presentation are predicated on mothers' risk factors, preferences, physical condition, and ability of the baby to breastfeed. Special attention is given to preserving the breastfeeding relationship.

Conclusion: This presentation calls attention to frequently overlooked lactation risk factors and suggests nursing interventions that can influence long-term maternal/infant outcomes.

\section{P24. Self-Care vs Self-Sacrifice in Medical-Surgical Nursing Culture: A Critical Ethnography}

Elise Juergens, BSN, RN, PhD-C

University of Southern Mississippi, Hattiesburg, MS

Background: Nursing continues to have ongoing retention challenges despite efforts to manipulate external causes. A literature search yielded scarce qualitative research on medical-surgical nurses and self-care. The purpose of this critical ethnography was to observe medical-surgical nursing culture related to self-care and self-sacrifice as reflected by behavior, dialog, and survey data.

Methods: Critical ethnography serves as the appropriate methodology based on research questions. Research participants are full-time, medical-surgical floor nurses working 12-hour day shifts at a large hospital in Mississippi. Observation, individual interviews, and the Nurse Codependency Questionnaire (NCQ) completion yielded raw data. The researcher spent 5 consecutive shifts with 10 participants.

Results: Research quantified concrete self-care behaviors such as nutrition, elimination, breaks, and hydration. Additionally, data analysis revealed several abstract self-care behaviors: support seeking, humor, expectation management, and authenticity. Six overarching themes emerged during data analysis: complaining, mistrust, inner conflict, collective caretaking, humor, and concrete self-care. Individual interview data corroborated observational data, and survey results triangulated findings. NCQ scores suggested minimal codependency among participants with a 0.92 Cronbach alpha reliability measure. Overall, the data suggest that cultural evolution is underway. Nurses adapt to strenuous environments and find new ways to engage in self-care.

Conclusion: This research provides a glimpse into the subculture of medical-surgical nursing. Nurses have long been portrayed as weary, hungry, or variations of the like. While this imagery is relatable, evolution has begun. Nurses have found a way to minimize self-sacrifice through collective self-care. In caring for each other, they cared for themselves. Improved cultural self-awareness can revise antiquated stereotypes. Moreover, improvements in nursing benefit healthcare organizations financially and with regard to patient care quality. Future research should seek to better understand other facets of nursing culture. Conceptual exploration in future research seeks to redefine what it means to be a nurse. 
P28. Using a Multidisciplinary Approach for the Reduction of Ventilator Hours in Coronary Artery Bypass Graft Sylvia Laudun, DNP, MBA, RN, CPHQ; Eugene Parrino, MD

Ochsner Medical Center, New Orleans, LA

Background: A review of isolated coronary artery bypass graft (CABG) surgery cases at an academic health system from 2013-2017 revealed total median ventilator times that were 2.2-3.6 hours greater than Society of Thoracic Surgeons (STS) data during the same period. Variation in the center's median total ventilator hours was noted, while STS revealed a consistent reduction over time.

Methods: In April 2017, a multidisciplinary team formed to design the ONE Path standardized care plan for CABG patients with the goal of improving care coordination and patient safety. Team meetings covered content development, electronic medical record testing, and implementation. The team reviewed retrospective data from the STS Adult Cardiac Surgery Database on total ventilator hours, extubation in the operating room (OR), initial intubation $<6$ hours, and reintubation events from 2016-2017 first quarter for isolated CABG patients. Cardiac surgeons met with cardiac anesthesiologists to propose methods to fast track appropriate patients in the OR and intensive care unit. In September 2017, implementation of ONE Path and changes in anesthesia OR practice occurred.

Results: Reduction in ventilation hours was essentially due to aggressive OR extubation and consistent care processes for early extubation. Pre-ONE Path implementation, 2017 median total ventilation time was 7.8 hours $(n=77)$ vs 0.0 hours $(n=74) 9$ months postimplementation of ONE Path and anesthesia changes. Extubation in the OR increased from $7.8 \%$ to $55.4 \%$, initial intubation $<6$ hours increased from $51.9 \%$ to $72.9 \%$, and reintubation rates dropped from $7.8 \%$ to $5.4 \%$.

Conclusion: A reduction in ventilation hours suggests the benefit of a multidisciplinary team approach to care coordination to improve outcomes.

P29. Measuring Direct Care Nurses' and Nurse Leaders' Perceptions of a Healthy Work Environment Within Acute Care Settings

Penny Huddleston, PhD, RN, NEA-BC, CCRN

Baylor Scott \& White Medical Center, Irving, TX

Background: Unhealthy work environments (UWEs) throughout healthcare organizations have been linked to absenteeism, ineffective delivery of healthcare to patients and families, high stress levels, poor communication, and ineffective collaboration and teamwork among healthcare professionals. Healthy work environments (HWE) lead to improved $\mathrm{RN}$ satisfaction.

Methods: These studies were conducted to replicate the findings of the original studies to reassess the psychometric properties of the Healthy Work Environment Scale for Direct Care Nurses (HWES for DCNs) and the Healthy Work Environment Scale for Nurse Leaders (HWES for NLs), to strengthen the generalizability of the tool and to describe the direct care nurses' perceptions of an HWE. Using nonexploratory descriptive designs, direct care nurses and nurse leaders were requested to complete the corresponding survey.

Results: Principal component analysis on the HWES for DCNs revealed 39 items with 6 components with eigenvalues higher than 1.0. The components were identified as genuine teamwork and true collaboration, meaningful recognition, appropriate staffing, effective decision-making, physical and psychological safety, and authentic leadership. The HWE standard of skilled communication loaded on all 6 of the components. The Cronbach alpha was 0.957. HWES for NLs revealed 40 items with 5 components with eigenvalues higher than 1.0. The components were identified as skilled communication, genuine teamwork and true collaboration, authentic leadership and effective decision-making, meaningful recognition, appropriate staffing, and physical and psychological safety. The Cronbach alpha was 0.974.

Conclusion: The HWES for DCNs and the HWES for NLs demonstrated strong validity and reliability to measure an HWE for nurses at all levels in acute care hospital settings. 
P30. Hiding in Plain Sight: An Innovative Hypertension Identification and Treatment Program

Louanne Friend, PhD, MN, RN; Suzanne Henson, MS, RD, LD

University of Alabama, Tuscaloosa, $A L$

Background: Hypertension (HTN) rates in Alabama are higher than the national average for all patients $\geq 45$ years, regardless of sex, race, geographic location (urbanity), or level of education. Nationwide, more than 7 million adults who are regularly accessing care and meet hypertensive criteria are not diagnosed or treated.

Methods: The Hiding in Plain Sight program, funded by a community grant from the Alabama Department of Public Health, was implemented at The University Medical Center (UMC), West Alabama's largest healthcare provider. The program was designed to identify patients with HTN using electronic health record templates and an evidence-based protocol. Institutional review board approval was obtained prior to data collection. Project dates were August 1, 2017June 29, 2018. Clinic training about the program, held in fall 2017, included all providers and nursing staff. Primary outcomes of this training included (1) implementing team huddles to identify patients with HTN and (2) standardizing blood pressure (BP) measurement in the clinic. The Alabama Hypertension Control Algorithm Template for Adults $\geq 18$ Years in the NextGen electronic medical record was rolled out February 1, 2018. The interprofessional research team also completed a patient education lifestyle training manual for UMC patients.

Results: We are capturing National Quality Forum 0018 metrics, a quality measure that records the percentage of patients 18-85 years of age who had a diagnosis of HTN and whose BP was adequately controlled $(<140 / 90 \mathrm{mmHg}$ ). The diagnosis of HTN has become a provider priority, and BP measurement and recording have become standardized throughout the clinic.

Conclusion: Electronic medical record space dedicated to HTN screening and treatment is an accomplishment and has made a difference in the culture at UMC Northport. We will be expanding the program to other UMC Tuscaloosa clinics and will collaborate with the College of Engineering to develop a lifestyle education app.

\title{
P33. 30 Minutes or Less: Closing the Gap Between Blood Glucose Check and Insulin Administration
}

\author{
Magally Rolen, BSN, RN, PCCN; Betsy Richter-Gifford, BSN, RN, CDE
}

Texas Health Resources, Arlington, TX

Background: Insulin is a high-risk medication with potential for serious harm or death. According to the Institute for Safe Medication Practices, insulin should be given within 30 minutes of blood glucose (BG) check. Meeting this standard in hospitals is a national problem. Audits revealed that our cardiac intermediate care unit was not consistently meeting the 30-minute standard.

Methods: A nurse champion designed and led the project. A new standardized unit process was created to perform BG checks at designated times and to administer insulin within 30 minutes of the BG check. Staff were educated on the evidence for change and recommended practice guidelines. Using chart audits, we provided consistent frequent feedback to staff, individually and as a group.

Results: Preintervention data was gathered from November 2017 and postintervention data from February-April 2018 , with an $n$ of 1,088 . BG checks done on time improved from $45 \%$ to $91.8 \%\left(I^{2}(1)=282.573, P<0.0001\right)$. The odds of BG checks being done on time were 8 times higher after the intervention. Insulin administration within the appropriate 30 -minute window improved from $33.1 \%$ to $82 \%\left({ }^{2}(1)=267.907, P<0.0001\right)$. The odds of insulin being given within 30 minutes were 9.2 times higher after the intervention. The time from BG check to insulin administration improved from 52.29 to 19.72 minutes after the project. This was a statistically significant improvement, $\mathrm{t}(631.617)=18.745$, $P<0.0001, \mathrm{~d}=1.19$.

Conclusion: It is vital to establish clear expectations for staff related to BG checks and insulin administration. Staff education and unit champions are critical to achieve goals in practice. Monitoring adherence through chart audits and providing individual feedback are key strategies to close the gap between BG checks and insulin administration. 


\section{P34. APRN vs RN: Evidence-Based Interventions to Treat Acute Pain in the Opioid Abuser}

Amy Holley, MSN, RN; Tyler Sturdivant, MSN, RN, AGCNS-BC

University of South Alabama, Mobile, AL

Background: With the increasing use of opioid medications to treat acute and chronic pain, the United States is facing an opioid abuse epidemic that significantly impacts healthcare. With the skyrocketing incidence of opioid abusers in the inpatient, outpatient, and community healthcare settings, nurses are tasked with centering the interprofessional team to appropriately care for these individuals. However, most nurses lack the skills, knowledge, and/or awareness about acute pain decision-making when they confront the opioid abuser.

Methods: The research question of "What evidence-based interventions are recommended for frontline registered nurses (RNs) and advanced-practice registered nurses (APRNs) when treating acute pain in the opioid abuse population?" guided literature exploration. A literature search was performed in CINAHL using a combination of keywords: nurse, opioid, abuse, pain, recommendations, and interventions. Articles were included if published within the last 5 years. Articles that discussed APRN and/or frontline RN recommendations were included. A total of 11 articles were selected, ranging from clinical practice guidelines to expert opinions.

Results: Recommendations for the frontline RN include providing education about nonpharmacologic pain relief measures to patients, consulting with a chaplain or a social services representative, evaluating medication administration, and communicating subjective and objective patient concerns to the healthcare provider. Recommendations for the APRN include prescribing specific nonpharmacologic and nonopioid options; following detailed opioid prescription guidelines; and referring patients to a psychologist, addictions specialist, and/or pain management specialist upon discharge.

Conclusion: Education of staff regarding evidence-based opioid treatment interventions will not only improve nursing competence but will also improve the quality of care provided to the growing opioid abuse population. 